\title{
Far-Infrared Ray Patch Non-Thermal Effects on Neck Pain Relief
}

\author{
Yi-Ching Cheng ${ }^{1,2}$, Ben-Yi Liau ${ }^{3}$, Ching-Lin Cheng4, Chi-Tang Ho ${ }^{5}$ and Yung-Sheng Lin ${ }^{6 *}$ \\ ${ }^{1}$ Department of Biological Science and Technology, College of Biopharmaceutical and Food Sciences, China Medical University, Taiwan \\ ${ }^{2}$ Linkwin Technology Co., LTD \\ ${ }^{3}$ Department of Biomedical Engineering, Hungkuang University, Taiwan
}

${ }^{4}$ Program of Digital Health Innovation, China Medical University, Taichung, Taiwan

${ }^{5}$ Department of Food Science, Rutgers University, New Brunswick, New Jersey, USA

${ }^{6}$ Department of Chemical Engineering, National United University, Taiwan

*Corresponding author: Yung-Sheng Lin, Department of Chemical Engineering, National United University, Miaoli, Taiwan.

Received Date: November 25, 2020

Published Date: December 03, 2020

\begin{abstract}
Modern people suffer from diseases of affluence such as neck disorders and other problem aches. They tend to use traditional therapies, but these therapies are often inconvenient. Far-infrared ray (FIR) therapy has been found to present good efficacy in relieving pain and solving problem aches without side effects. FIR has many bio-effects including thermal and non-thermal effects. Previous studies usually focus on the thermal effect. This study examines the non-thermal effects of a far-infrared ray patch. The experimental and control groups used patches with and without FIR, respectively. Forty adults with neck discomfort were randomly divided into two equally populated parallel trials. The FIR patch could significantly enhance the neck temperature by $1.7^{\circ} \mathrm{C}$, but the control group saw an increase of $1.3^{\circ} \mathrm{C}$. The FIR patch significantly improved blood flow to $109.7 \%$, which was significantly better than the $99.6 \%$ in the control. The heart rate variability increased by $22 \%$ and $19.6 \%$ in the experimental and control groups, respectively. As for the visual analog index, the experimental group expressed significant reduction in pain and anxiety levels. These results indicated that the FIR patch non-thermal effects produced good results in improving neck discomfort symptoms.
\end{abstract}

Keywords: Far-infrared ray; Neck pain; Non-thermal effect

\section{Introduction}

Modern people suffer from diseases of affluence such as neck disorders and other problem aches caused by overwork, incorrect sitting position and pressure [1]. People feel physically ill and mentally anxious, often experiencing disturbing pain throughout the day and night. Symptoms such as anorexia or insomnia can cause the patient to suffer from lack of nourishment, lowered functional immunity and sickness [2].

The uncomfortable symptoms from this type of neck disorder can be relieved by taking nonsteroidal anti-inflammatory drugs or a muscle relaxant. However, overusing medicine could lead to side effects such as gastrointestinal discomfort, dizziness and nausea [3]. Even worse, drug overuse could result in insensitivity, leading to chronic pain. Furthermore, prolonged drug use will increase the potential for side effects.

Consequently, developing a complementary therapy free from drugs and their side effects is worthwhile. There are traditional complementary therapies like acupuncture [4], massage, joint stretching, aromatherapy [5], strength exercises [6] and Qigong [7] used to make the neck disorder better. All of these conventional therapies require a professional to perform the necessary therapy. 
FIR has many bio-effects including thermal effects and non-thermal effects [8-10]. FIR has generally been evaluated as an effective, safe and non-pharmacologic alternative for pain relief. The biological benefits of FIR have been studied over the past 2 decades. FIR operates at a wavelength ranging between 4 and $14 \mu \mathrm{m}$ [11-13]. FIR can activate both thermal and non-thermal effects at the molecular level to influence the health of a whole living organism [14]. The versatile beneficial effects and the mechanism of FIR on pain relief and mental disorders remain less understood; especially the nonthermal FIR effect. A high interest exists in these aspects to clarify the efficacy of FIR therapy.

Many studies examined FIR therapy as a complementary treatment. Even though it has been proven effective in healing wounds, relieving pain, ameliorating fatigue and improving mood $[15,16]$, the evidence was limited to self-rating evaluations with no objective physical evidence.

Most studies emphasized only the FIR thermal effect with the non-thermal effect not examined. A study claimed that the nonthermal effect on endothelial cell anti-apoptosis may contribute to vascular access [17]. Because FIR bio-effects consist of thermal and non-thermal effects, the nonthermal effect must be discussed independently. The study was aimed at determining the FIR nonthermal effect on neck pain relief. We evaluated patient self-ratings on pain and anxiety and provide objective physical evidence including warming capability, blood flow and heart rate variability. This study provides valuable information on the non-thermal FIR benefits for neck disorder sufferers who may use the FIR patch in the future.

\section{Material and Methods}

\section{Participants}

This clinical trial was approved by the independent ethics committee of China Medical University, with certification by the Institutional Review Board (IRB) approval number DMR101IRBI-219. The study volunteers gave legal consent to participate in this clinical trial and were aged18 to 40 years. There were 40 participants who were randomly divided to a control group(the patch without FIR, $n=20$ ) and the experimental group (the FIR patch, $n=20$ ).

\section{Patches}

Two different patches were used to examine the non-thermal FIR effect. The control group used a patch (ThermaCare ${ }^{\circledR}$ Neck, Wrist \& Shoulder) without FIR imported from America (Figure 1). The experimental group used the FIR carbon fiber patch obtained from Linkwin Technology Co., LTD. (Figure 2). The experimental data were recorded as skin temperature, blood flow and heart rate variability.

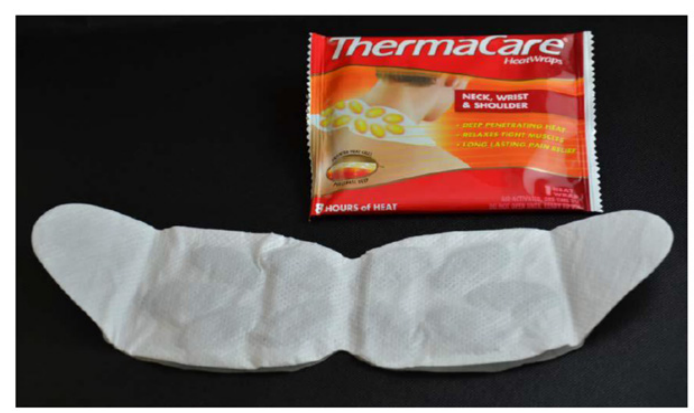

Figure 1: ThermaCare ${ }^{\circledR}$ Neck, Wrist \& Shoulder patch. The size is $19 \mathrm{~cm} \times 7.5 \mathrm{~cm}$.

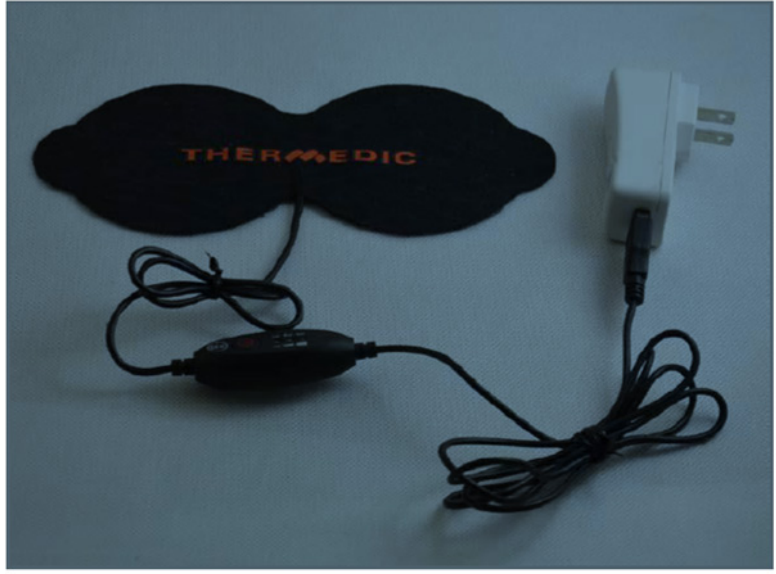

Figure 1: ThermaCare ${ }^{\circledR}$ Neck, Wrist \& Shoulder patch. The size is $19 \mathrm{~cm} \times 7.5 \mathrm{~cm}$. 


\section{Skin temperature and blood flow}

All measurements and procedures in this study were carried out in a limate-controlled room at a constant temperature and humidity (23 ${ }^{\circ} \mathrm{C}$ and $60 \%$ relative humidity) [18]. Before assessment the participants were required to sit in a chair in the aforementioned room. Participants were thermal graphed to measure the neck area temperature using a Fluke Ti25 thermal imager (Fluke Corporation, Everett, WA, USA). Their neck blood flow was detected using a MoorLDI2-IR Laser Doppler Imager (Moor Instruments Ltd., Devon, UK) as described in the previous report [19].

\section{Heart rate variability}

This study used heart rate variability (HRV) as an experimental parameter to test FIR efficacy. HRV was measured using ANSWatch TS-0411 (Taiwan Scientific Corp., Taiwan, Republic of China) [20]. The participants sat on the chair, put on the detector around their left arm, closed their eyes and stayed calm while HRV was measured [18].

\section{Visual analog scale}

Psychometric measurements were taken to assess the participants' pain intensity and anxiety. These tests were assessed using the standard visual analogue scale (VAS).

VAS is a common clinically used evaluation for assessing pain and anxiety [21-24]. Participants were asked to place a mark on a $10-\mathrm{cm}$ horizontal line to express how much pain they felt. The left-end indicated no pain and the right-end indicated severe pain.

\section{Statistical analysis}

All data between groups before and after using patches were analyzed using the Wilcoxon Signed- Rank Test using the SPSS 12.0 software package for statistical analysis. A value of $\mathrm{P}<0.05$ was regarded as statistically significant (*).

\section{Result and Discussion}

\section{The heating rates and skin temperature of the fat- infrared patch}

According to Figure 3 the initial temperatures of the two studied patches were equal at $23{ }^{\circ} \mathrm{C}$. After a 30-minute heating process the temperature of the control and experimental group patches were $34.4 \pm 0.4{ }^{\circ} \mathrm{C}$ and $34.1 \pm 0.5{ }^{\circ} \mathrm{C}$, respectively. The dynamic temperature changes in the heating process indicated that the temperature of two patches were not significantly different. These two patches therefore served the materials for studying the FIR non-thermal effect. According to Figure 4, the beginning skin temperature was $33.7 \pm 0.2{ }^{\circ} \mathrm{C}$ for the control group. After using the patch for 30 minutes the neck skin temperature increased significantly to $34.9 \pm 0.2{ }^{\circ} \mathrm{C}(\mathrm{p}<0.05)$.

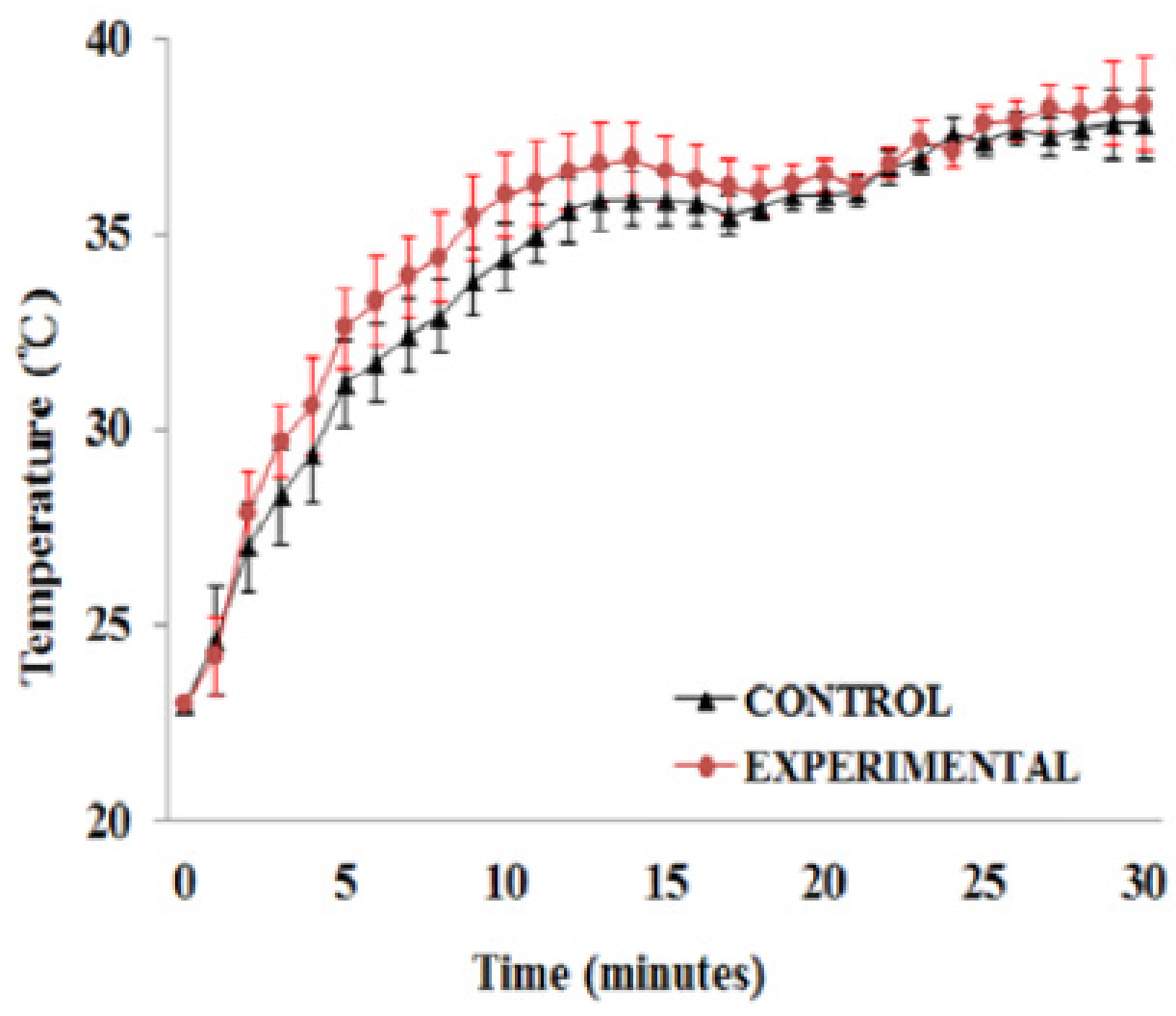

Figure 3: Comparison of elevated temperature in two groups within 30 minutes. 


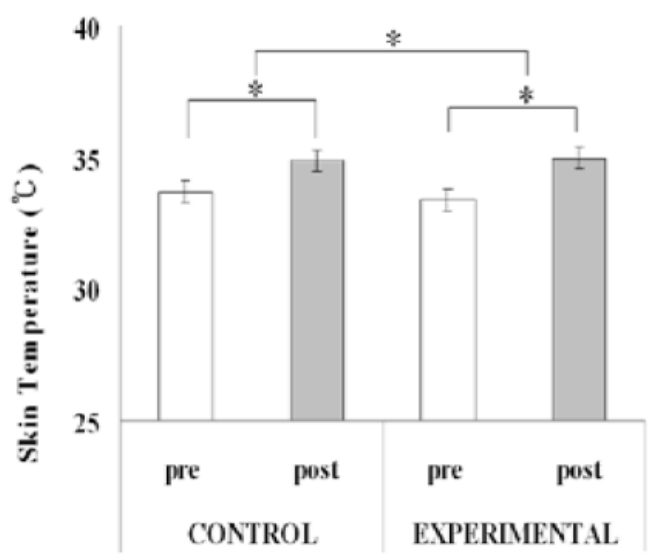

Figure 4: Comparison of skin temperature in two groups before and after a patch treatment. The symbol '*' indicates a significant difference between groups using the Wilcoxon Signed-Rank Test $(p<0.05)$.

The beginning skin temperature of the experimental group was $33.4 \pm 0.1{ }^{\circ} \mathrm{C}$. Under the same environmental conditions, the neck temperature increased to $35.1 \pm 0.1^{\circ} \mathrm{C}(\mathrm{p}<0.05)$. After applying the patches, the neck temperatures in the two groups both tangibly increased. Comparing the elevated neck temperature in the two groups we found that the FIR patch $\left(1.2^{\circ} \mathrm{C}\right.$ increase) was significantly higher than the control group $\left(1.7^{\circ} \mathrm{C}\right.$ increase). Hot devices provided thermal energy in this study and both groups experienced this effect in elevating skin temperature. It is known that a patch or heat device with FIR can keep the body warm. Our evidence further demonstrated that the FIR patch could produce a higher temperature than a patch without FIR. This represents that the FIR non-thermal effect could contribute an increase in skin temperature.

\section{The blood flow improvement of the far-infrared patch}

Before wearing the patch, the control group blood flow was measured by the perfusion unit (PU) as 76.4 \pm 4.8 PU. According to Figure 5 after the patch acted for 30 minutes the blood flow increased significantly to $152.5 \pm 12.1 \mathrm{PU}(\mathrm{p}<0.05)$. Before wearing the patch, the experimental group blood flow was measured by the perfusion unit as $79.7 \pm 6$ PU. Under the same conditions the blood flow increased significantly to $167.2 \pm 15.1 \mathrm{PU}(\mathrm{p}<0.05)$. After the two groups used the patches, the neck blood flow increased. Comparing the neck blood flow for the two groups we found that the experimental group showed higher blood flow than the control group $(\mathrm{p}<0.05)$. The blood flow of the control group increased 99.6\%, while the experimental group increased $109.7 \%$. An increase in blood flow indicated rapid hemodynamic changes and an increasing volume of blood in the neck region. When blood circulation was improved it could quickly expel lactic acid, inflammation and toxin extravasations.

These substances are considered to affect muscle cells and neural pathways and cause pain $[25,26]$. The data from this study demonstrated that the non-thermal FIR patch increased blood flow better than the thermal FIR patch.

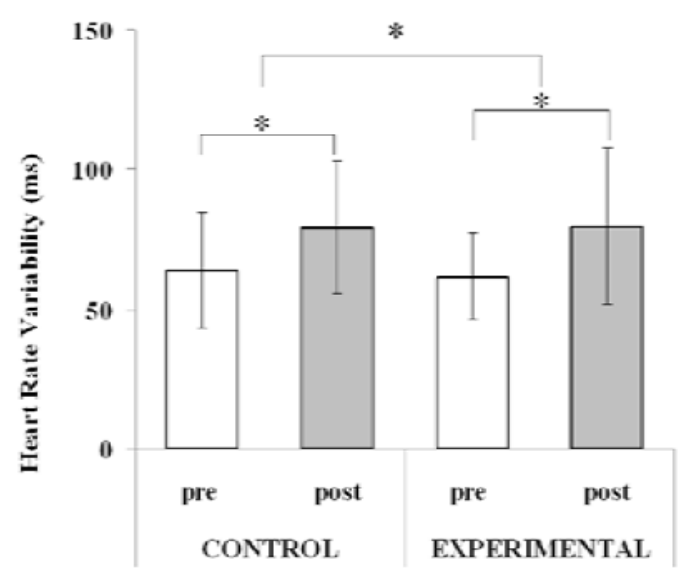

Figure 5: Comparison of heart rate variability in two groups before and after a patch treatment. The symbol '*' has a significant difference between groups using the Wilcoxon Signed-Rank Test $(p<0.05)$. 


\section{The autonomic nervous system improvement of the far- infrared patch}

HRV is a valid biomarker for assessing the autonomic nervous system function [27]. The heart rate variability of the control group before wearing the patch was $63.9 \pm 20.9 \mathrm{mini}$ second(ms) (Figure 6). After 30 minutes the HRV of the control group increased significantly to $79.5 \pm 23.8 \mathrm{~ms}$ ( $\mathrm{p}<0.05$ ). The HRV of the experimental group before wearing the patch was $61.7 \pm 22$.0ms. Under the same conditions the heart rate variability of the experimental group increased to $79.8 \pm 24.9 \mathrm{~ms}$. The HRV of the control group increased approximately $19.6 \%$ and the experimental group increased by about $22.6 \%$.HRV is a neuro-control factor in the heart and has used more in recent scientific research in evidence-based traditional medicine. The HRV rate generally increases with parasympathetic activity. The parasympathetic nervous system is associated with the pain relief process. With parasympathetic predominance, vasodilatation is aroused to improve peripheral blood flow and promote blood circulation to alleviate muscle pain and decrease mood disorders though muscle tension relief [28]. This paper found that the FIR group exhibited a significant effect on autonomic nervous system regulation.

\section{Efficacy of reducing pain and anxiety from far-infrared patch}

Neck muscle tension is associated with mental stress and once neck muscle rigidity is alleviated anxiety diminishes [29,30]. Figure 7 indicates the outcomes from pain and anxiety alteration from applying the patch from start to finish. The results show that the two groups exhibited decreased VAS from the patch application to the neck, reflecting the two different patches have efficacy in improving anxiety. Furthermore, the experimental group demonstrated better efficacy than the control group. Previous studies demonstrated that a thermal effect relieves pain and decreases anxiety [31,32]. This study showed that extraordinary outcome. The non-thermal effect patch reflected better satisfactory evidences in pain relief and anxiety reduction than the thermal patch. This indicated that the non-thermal effect could provide better therapy for pain and anxiety. The pain is characterized by bio-molecular induction of cyclooxygenase(COX-2) and prostaglandin E2. The FIR therapy could inhibit the induction of these two pain related biomolecules to relive pain [33]. Furthermore, the acute increase in nitric oxide production by FIR may also help to reduce the pain [34,35].

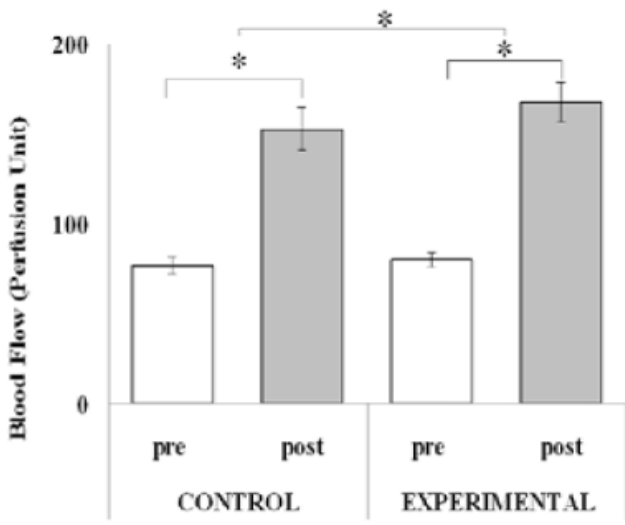

Figure 6: Comparison of blood flow in two groups before and after a patch treatment. The symbol '*' indicates a significant difference between groups using the Wilcoxon Signed-Rank Test $(p<0.05)$.

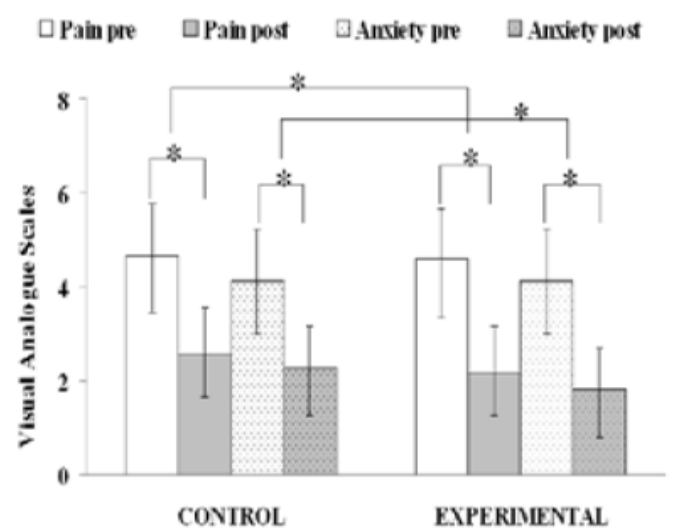

Figure 7: Comparison of pain and anxiety in two groups before and after a patch treatment. The symbol '*' has a statistical difference between groups using the Wilcoxon Signed-Rank Test $(p<0.05)$. 


\section{Limitations}

This study demonstrated that the non-thermal effects of the FIR patch can significantly improve neck disorders. However, the improvement was observed only for a short-term application and the pain relief effect was measured immediately after intervention. The findings of our experiment were in agreement with those of a prior study. A follow-up protocol for long-term FIR application is necessary to evaluate the long-term effects in improving neck pain. Our investigation revealed that after short-term use the FIR patches immediately demonstrated the capacity to reduce anxiety. This reflects that when consumers select such products to improve neck disorders they may be satisfied with the warm/thermal effect after a short period.

Studies providing more physical evidence of neck disorder relief and identification of the biological benefits felt by the participants is required for future FIR patch development.

\section{Conclusion}

This study verified the FIR non-thermal neck pain relief effect. Compared to the control group without FIR, the FIR patch demonstrated brilliant improvements in skin warming capability, blood flow, heart rate variability and pain and anxiety reduction. This innovative FIR patch can provide complementary therapy to help solve neck pain disorders. This work was just a short-term experiment to show the advantages of FIR. A long-term study to evaluate the non-thermal effect on neck disorder relief should be carried out.

\section{Summary}

This study verified the FIR non-thermal neck pain relief effect. Compared to the control group without FIR, the FIR patch demonstrated brilliant improvements in skin warming capability, blood flow, heart rate variability and pain and anxiety reduction This innovative FIR patch can provide complementary therapy to help solve neck pain disorders. This work was just a short-term experiment to show the advantages of FIR. A long-term study to evaluate the non-thermal effect on neck disorder relief should be carried out.

\section{Acknowledgement}

This research project was sponsored by Taiwan National Science and Technology Committee of the Administrative Academy with Grant number 101WFDA900046.

\section{Conflict of Interest}

Authors declare no conflict of interest.

\section{References}

1. Hoy DG, Protani M, De R, Buchbinder R (2010) The epidemiology of neck pain. Best Pract Res Clin Rheumatol 24(6): 783-792.

2. Palermo TM, Law E, Churchill SS, Walker A (2012) Longitudinal course and impact of insomnia symptoms in adolescents with and without chronic pain. J Pain 13(11): 1099-1106.
3. Van der Velde G, Hogg-Johnson S, Bayoumi AM, Cassidy JD, Côté P, et al. (2008) Identifying the best treatment among common nonsurgical neck pain treatments. Euro Spine J 17: 184-191.

4. Chen HM, Chen CH (2004) Effects of acupressure at the Sanyinjiao point on primary dysmenorrhoea. J Adv Nur 48(4): 380-387.

5. Han SH, Hur MH, Buckle J, Choi J, Lee MS (2006) Effect of aromatherapy on symptoms of dysmenorrhea in college students: a randomized placebo-controlled clinical trial. J Altern Complem Med 12(6): 535-541.

6. Furlan AD, Yazdi F, Tsertsvadze A, Gross A, Van Tulder M, et al. (2012) A Systematic Review and Meta-Analysis of Efficacy, Cost-Effectiveness, and Safety of Selected Complementary and Alternative Medicine for Neck and Low-Back Pain. Evid-Based Compl Alt 2012: 953139.

7. Von Trott P, Wiedemann AM, Lüdtke R, Reißhauer A, Willich SN, et al. (2009) Qigong and exercise therapy for elderly patients with chronic neck pain (QIBANE): a randomized controlled study. J Pain 10(5): 501508

8. Leung TK, Lin YS, Lee CM, Chen YC, Shang HF, et al. (2011) Direct and indirect effects of ceramic far infrared radiation on the hydrogen peroxide-scavenging capacity and on murine macrophages under oxidative stress. J Med Biol Eng 31: 345-351.

9. Leung TK, Shang HF, Chen DC, Chen JY, Chang TM, et al. (2011) Effects of far infrared rays on hydrogen peroxide-scavenging capacity. Biomed Eng Appl Basis Comm 23: 99-105.

10. Leung TK, Chan CF, Lai PS, Yang CH, Hsu CY, et al. (2012) Inhibitory effects of far-infrared irradiation generated by ceramic material on murine melanoma cell growth. Int J Photoener 2012: 1-8.

11. Leung TK, Lee CM, Lin MY, Ho YS, Chen CS, et al. (2009) Far infrared ray irradiation induces intracellular generation of nitric oxide in breast cancer cells. J Med Biol Eng 29: 15-18.

12. Leung TK, Lin YS, Chen YC, Shang HF, Lee YH, et al. (2009) Immunomodulatory effects of far infrared ray irradiation via increasing calmodulin and nitric oxide production in RAW 264.7 macrophages. Biomed Eng Appl Basis Comm 21: 317-323.

13. Ishibashi J, Yamashita K, Ishikawa T, Hosokawa H, Sumida K, et al. (2008) The effects inhibiting the proliferation of cancer cells by far-infrared radiation (FIR) are controlled by the basal expression level of heat shock protein (HSP) 70A. Med Oncol 25(2): 229-237.

14. Hsu YH, Chen YC, Chen TH, Sue YM, Cheng TH, et al. (2012) Far-infrared therapy induces the nuclear translocation of PLZF which inhibits VEGFinduced proliferation in human umbilical vein endothelial cells. Plos One 7(1): e30674.

15. Matsumoto S, Shimodozono M, Etoh S, Miyata R, Kawahira K (2011) Effects of thermal therapy combining sauna therapy and underwater exercise in patients with fibromyalgia. Compl Ther Clin Pract 17(3): 162-166.

16. Ke YM, Ou MC, Ho CK, Lin YS, Liu HY, et al. (2012) Effects of somatothermal farinfrared ray on primary dysmenorrhea: a pilot study. EvidBased Compl Alt 2012: 240314

17. Ko WS, Chen TH, Chen CH, Chen TW, Chen YC (2012) Non-thermal effect of far-infrared activates Akt to reduce doxorubicin-induced apoptosis in human umbilical vein endothelialcells. Acta Nephrol 26: 2-7.

18. Hug AM, Schmidts T, Kuhlmann J, Segger D, Fotopoulos G, et al. (2011) Skin hydration and cooling effect produced by the Voltaren vehicle gel. Skin Res Technol 18(2): 199-206.

19. West XZ, Malinin NL, Merkulova AA (2010) Oxidative stress induces angiogenesis by activating TLR2 with novel endogenous ligands. Nature 467(7318): 972-976.

20. Chang KM, Shen CW (2011) Aromatherapy benefits autonomic nervous system regulation for elementary school faculty in Taiwan. Evid-Based Compl Alt 2011: 946537.

21. Lee CH, Roh JW, Lim CY, Hong JH, Lee JK, et al. (2011) A multicenter randomized, doubleblind, placebo-controlled trial evaluating the 
efficacy and safety of a far infrared-emitting sericite belt in patients with primary dysmenorrheal. Complement Ther Med 19(4): 1871-193.

22. Masuda A, Kihara T, Fukudome T, Shinsato T, Minagoe S, et al. (2005) The effects of repeated thermal therapy for two patients with chronic fatigue syndrome. J Psychosom Res 58(4): 383-387.

23. Beever R (2010) The effects of repeated thermal therapy on quality of life in patients with type II diabetes mellitus. J Altern Complement Med 16(6): 677-681.

24. Kane RL, Bershadsky B, Rockwood T, Saleh K, Islam NC (2005) Visual Analog Scale pain reporting was standardized. J Clin Epidemiol 58(6): 618-623.

25. Lauche R, Cramer H, Hohmann C, Choi KE, Rampp T, et al. (2012) The effect of traditional cupping on pain and mechanical thresholds in patients with chronic nonspecific neck pain: A randomized controlled pilot study. Evid-Based Compl Alt 2012: 429718.

26. Lee YH, Park BNR, Kim SH (2011) The effects of heat and massage application on autonomic nervous system. Yonsei Med J 52(6): 982-989.

27. Matsubara T, Ara YCP, Shiro Y, Shimo K, Nishihara M, et al. (2011) Comparative effects of acupressure at local and distal acupuncture points on pain c conditions and autonomic function in females with chronic neck pain. Evid-Based Compl Alt 2011: 543291.

28. Nagashima Y, Igaki M, Suzuki A, Tsuchiya S, Yamazaki Y, et al. (2011) Application of a heat- and steam-generating sheet increases peripheral blood flow and induces parasympathetic predominance. Evid-Based Compl Alt 2011: 965095.
29. Matsui T, Fujimoto T (2011) Treatment for depression with chronic neck pain completely cured in $94.2 \%$ of patients following neck muscle treatment. Neurosci Med 2: 71-77.

30. Mäntyselkä P, Lupsakko T, Kautiainen H, Vanhala M (2010) Neckshoulder pain and depressive symptoms: a cohort study with a 7-year follow-up. Eur J Pain 14(2): 189-193.

31. Pan SY, Gao SH, Zhou SF, Tang MK, Yu ZL, et al. (2012) New perspectives on complementary and alternative medicine: an overview and alternative therapy. Altern Ther Health Med 18(4): 20-36.

32. Wong CH, Lin LC, Lee HH, Liu CF (2012) The analgesic effect of thermal therapy after total knee arthroplasty. J Alt Complement Med 18(2): 175179.

33. Leung TK, Liu YC, Chen CH, Hsieh NF, Chen KC, et al. (2013) In vitro cell study of possible anti-inflammatory and pain relief mechanism of farinfrared ray-emitting ceramic material. J Med Bio Eng 33: 179-184.

34. Liau BY, Leung TK, Ou MC, Ho CK, Yang AG, et al. (2012) Inhibitory effects of far-infrared ray emitting belts on primary dysmenorrheal. Int J Photoener 2012: 238468.

35. Park JH, Lee S, Cho DH, Park YM, Kang DH, et al. (2013) Far-infrared radiation acutely increases nitric oxide production by increasing $\mathrm{Ca}^{2+}$ mobilization and $\mathrm{Ca}^{2+} /$ calmodulin-dependent protein kinase IImediated phosphorylation of endothelial nitric oxide synthase at serine 1179. Biochem Biophys Res Commun 436(4): 601-606. 